\title{
Studying the transition from light emitting diodes to semiconductor lasers in applied physics laboratories
}

\section{K.-P. Möllmann, M. Regehly, M. Vollmer}

K.-P. Möllmann, M. Regehly, M. Vollmer, "Studying the transition from light emitting diodes to semiconductor lasers in applied physics laboratories," Proc. SPIE 11143, Fifteenth Conference on Education and Training in Optics and Photonics: ETOP 2019, 1114312 (2 July 2019); doi: 10.1117/12.2523387 Photonics: ETOP 2019, 2019, Quebec City, Quebec, Canada 


\title{
Studying the transition from light emitting diodes to semiconductor lasers in physics laboratories
}

\author{
K.-P. Möllmann, M. Regehly, M. Vollmer* \\ University of Applied Sciences Brandenburg, Magdeburgerstr. 50, 14770 Brandenburg, Germany
}

\begin{abstract}
Light emitting diodes and semiconductor lasers belong to almost any curriculum in the field of physics, optics or photonics programs at universities. The physics concepts are usually introduced in Bachelor programs, but a real deep understanding which is also based on laboratory experiments is seldomly acquired before studying in Master programs. Here we present some laboratory experiments for students of a photonics Master program at our University of Applied Sciences. Students are required to study the transitions from LEDs to semiconductor lasers in a four step investigation. First they record the diode characteristic of output power versus current. This helps to identify the pure LED regime, the transition region and the pure laser regime of the devices. Second they study the spatial characteristics of the commercial LED / laser devices with or without additional lenses. Third, laser dimensions are measured with microscopy and fourth the changes of the emissions spectra while increasing the injection current from LED to laser are measured with high accuracy using a Fourier Transform Infrared spectrometer. Here we present and discuss exemplary results of the first two steps and briefly mention the subsequent ones.
\end{abstract}

Keywords: Optics education, semiconductor lasers, output characteristics, beam profile, microscopy, FTIR spectroscopy

\section{INTRODUCTION}

The invention of the laser revolutionized the field of optics and quantum physics. Similarly, the advancements in semiconductor and microsystem technologies lead to substantial improvements of semiconductor diode lasers (e.g. [1,2]) which - due to the microscopic dimensions opened up many new application fields in photonics. The present paper presents a selection of experiments designed as part of a student laboratory course for photonics master programs. Some of them use standard equipment while others require special apparatus usually available in many university based research labs.

Nowadays, laser diodes are fabricated in a broad variety of different designs ranging from simple heterostructures to quantum well and quantum dot systems. Nearly all emission wavelengths from the UV to the thermal IR are meanwhile available. Concerning geometry, one may purchase anything from simple and most common strip lasers to more complex vertical cavity lasers. Due to the advancement of microsystems, massive scale fabrication has become inexpensive. Cheapest laser diodes including already a collimation lens are available for around $10 \mathrm{cts}$ per piece in packages of 10 or even 100 laser modules. Fig. 1 shows photos of such diodes as bought and after the lens had been removed.

These systems are hence even privately accessible for anyone interested, however, the physics behind is often more complex than anticipated. For this reason we have developed a series of laboratory experiments based on such inexpensive laser diodes with the goal of a more or less complete characterization concerning their electrical and optical properties. The lab course takes place after students have learned about the physics of diode lasers which will not be discussed here. We also assume that students know that inexpensive lasers operate using cleaving facets with typical refractive index of $\mathrm{n} \approx 3.6$, i.e. reflectivities $R=\frac{(n-1)^{2}}{(n+1)^{2}}$ of around $32 \%$ as "mirrors".

Students are separated into groups which perform the following tasks with laser diodes from the same batch.

*vollmer@th-brandenburg.de; phone +49 3381355347

Fifteenth Conference on Education and Training in Optics and Photonics: ETOP 2019, edited by

Anne-Sophie Poulin-Girard, Joseph A. Shaw, Proc. of SPIE Vol. 11143, 1114312 · (c) 2019

SPIE, ICO, IEEE, OSA · CCC code: 0277-786X/19/\$18 · doi: 10.1117/12.2523387

Proc. of SPIE Vol. $111431114312-1$ 


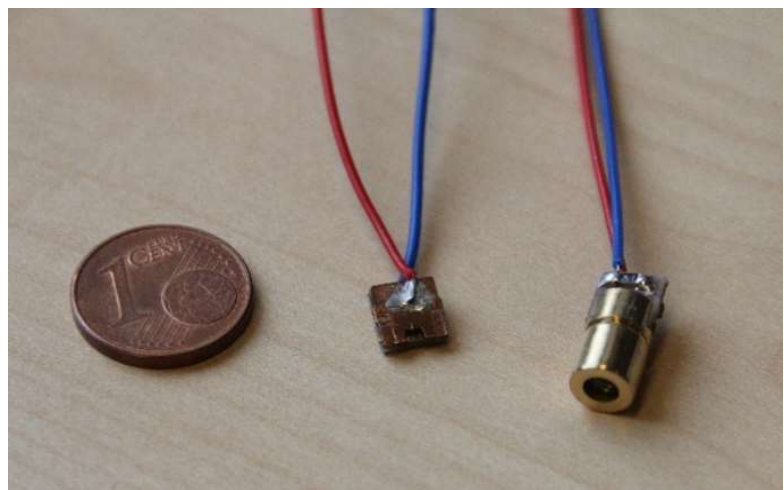

Figure 1. Commercially available inexensive laser diodes with prices of around $10 \mathrm{ct}$ per piece. After removing the lens the whole assembly has dimensions of $5 \mathrm{~mm}$ by $5 \mathrm{~mm}$ by $1 \mathrm{~mm}$.

1) Electrical characterization of the diodes
a) current vs. voltage

b) power vs. current

2) Spatial beam profiles

a) during transition from LED emission to laser mode without lens

b) laser far field pattern with lens

3) Visual (and maybe electron) microscopic inspection of the diode laser

a) quantitative analysis of laser dimensions

b) observation of laser during operation

4) High resolution spectroscopic characterization of the output of the diode

a) center wavelength and FWHM

b) longitudinal modes

After their measurements, students gather to present and discuss their results. They combine all data into a single presentation for the $\mathrm{TA} /$ professor and submit a respective detailed analysis with the complete characterization of the laser.

The attractiveness of the tutorial lies on one side in the exploration of the visual phenomena (from $2 \mathrm{a}$ and $3 \mathrm{~b}$ ) and on the other side in trying to quantitatively understand the experiments ( $3 \mathrm{a}$ and $4 \mathrm{~b}$ ) by connecting them to the theory.

Of course, characterization of laser diodes belongs to the curriculum of many physics, optics and / or photonics programs, and respective lab courses are widely available. The standard set usually includes studying $\mathrm{I}(\mathrm{U})$ and $\mathrm{P}(\mathrm{I})$ characteristics and often integrate qualitative beam profile measurements by scanning across the beam using a pinhole or by recording it with cameras. Sometimes even beam quality parameters like $\mathrm{M}^{2}$ are evaluated. In addition, many courses include spectral analysis, however, mostly done using low resolution grating spectrometers. Our new lab course on laser diode characterization also addresses these common topics of recording the characteristics I(U) and P(I) curves, as well as beam profiles with a camera. In addition, however, microscopy is used to determine cavity lengths and dimensions of the lasers and even perform microscopic in situ observations while operating the laser. Finally, high resolution Fourier transform spectrometry allows to observe individual longitudinal modes and quantitatively correlate cavity length with observed longitudinal mode spacing.

In this work we discuss exemplary results of the electrical characterization and beam profiles of the laser diodes. The microscopy and spectroscopy experiments and analysis are just briefly mentioned, they will be discussed in detail elsewhere [3]. 


\section{ELECTRICAL CHARACTERIZATION OF LASER DIODES}

Laser diodes are first of all semiconductor diodes and should behave as such. From textbooks, one usually expects the following exponential ideal I(U) characteristic of any diode, also known as the ideal Shockley diode equation:

$$
I(U)=I_{0}\left(\exp \frac{e U}{k T}-1\right)
$$

where $\mathrm{I}_{0}$ is the reverse bias saturation current. In practice, such a behavior is nearly never found for LEDs or semiconductor lasers. Rather a more or less linear current vs. voltage characteristic is usual across most of the operational region (as can also be seen in our recorded characteristics, see below). Students usually have the misconception that it only looks linear but in reality is still exponential. However, students could and should explain the discrepancy as due to the neglect of any resistance in Eq. 1.

Any injection diode has an overall resistance $\mathrm{R}_{\mathrm{S}}$ which is due to three contributions in series: the resistances of the $\mathrm{p}$ region, the one of the space charge region and the one of the n-region. As first approximation, one usually neglects any resistance and the Shockley special case of diffusion limited currents then gives Eq. 1.

We note that Eq. 1 may be improved by taking into account that the transition is not abrupt and that currents are not limited by diffusion, but are governed by recombination and generation. Therefore a factor $m>1$ is usually introduced in the exponent

$$
I=I_{0}\left(\exp \frac{e U}{m k T}-1\right)
$$

For simplicity we assume $\mathrm{m}=1$ in the following.

For any real diode, however, the resistance of the $\mathrm{p}$ and $\mathrm{n}$ regions must also be taken into account (for typical voltages in forward direction, the resistance of the space charge region is much smaller). Due to the voltage drop in the contacting regions and often also an external resistor the characteristic is described by:

$$
I=I_{0}\left[\exp \left(\frac{e\left(U-I R_{S}\right)}{k T}\right)-1\right]
$$

where $\mathrm{R}_{\mathrm{S}}$ describes the overall series resistance. Solving Eq. 3 for $\mathrm{U}$ gives

$$
\left.U=\frac{k T}{e} \ln \left(\frac{I}{I_{0}}+1\right)\right)+I R_{S}
$$

which describes the behavior of the expected observable I(U) characteristic much better than Eq. 1.

One may also differentiate Eq. 4 to find the differential resistance:

$$
R_{\text {diff }}=\frac{d U}{d I}=\frac{k T}{e I_{0}} \exp \left(-\frac{e\left(U-I R_{S}\right)}{k T}\right)+R_{S}
$$

As $\mathrm{kT} / \mathrm{e}$ at room temperature amounts to about $25 \mathrm{mV}$, it is obvious, that for large values of ( $\mathrm{U}-\mathrm{I} \mathrm{R}_{\mathrm{s}}$ ) above $1 \mathrm{~V}$ (which is typical for laser operation), the exponential is of the order of $10^{-18}$. Even if the reverse bias saturation current would be very small of the order of $1 \mathrm{pA}$, the differential resistance is dominated by the series resistance, which can be of the order of $100 \Omega$. As a consequence, the initially nonlinear increase of current with voltage soon leads to the observed more or less linear $\mathrm{I}(\mathrm{U})$ characteristic.

In order to verify the expected linear dependency, the first task of students is to record current I(U) and optical power output P(I) characteristics of at least one diode at room temperature $(293 \mathrm{~K})$. In order to keep the tasks manageable in the allocated lab time, we do not discuss temperature variation effects.

Fig. 2a shows the results I(U) for one arbitrarily selected laser diode with added linear fit for the laser operation mode. Fig. $2 \mathrm{~b}$ shows the same for P(I). Both plots closely follow a linear behavior above threshold. 

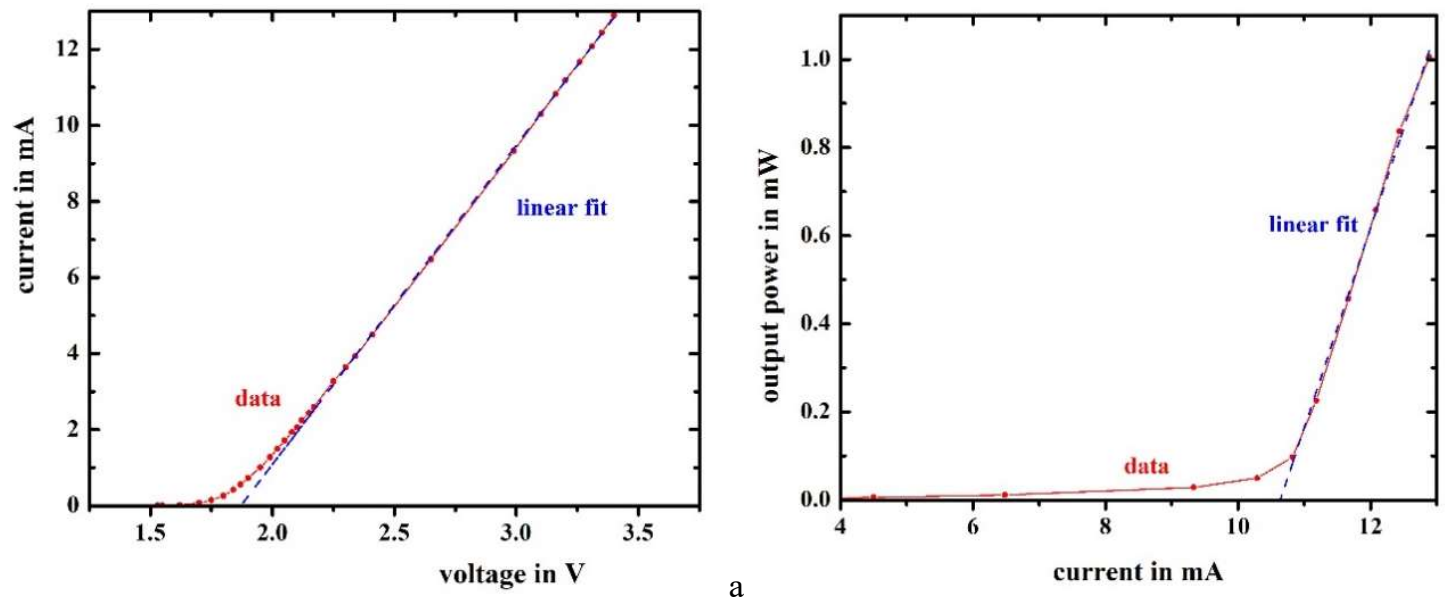

Figure 2. a) Current vs voltage characteristics of a specific laser diode at room temperature (293K). The threshold voltage, given by the intercept of the linear fit in the lasing regime with the voltage axis, is $1.88 \mathrm{~V}$. b) Power versus current characteristic of the same commercial laser diode. The threshold current, given by the intercept of the linear fit in the lasing regime with the current axis, is $10.63 \mathrm{~mA}$.

Students often question why -similar to I(U) - the optical output P(I) shows a linear dependence with injection current as well. Reason is that with increasing injection current, there is a constant fraction of injected electrons, which lead to recombination via light emission. Of course, this only applies to some maximum current as the diode also suffers from the large thermal load, which may ultimately destroy the device (efficiencies of LEDS and lasers can reach up to $50 \%$, but often they are operated with unfavorable parameters, which means that - as in the case of our semiconductor lasers - more than $90 \%$ of the electrical input power leads to thermal losses).

The P(I) characteristic exhibits the LED and the laser region. The different slopes can be due first to the measurement technique: the optical output is measured in the forward direction. The LED emits more or less isotropically, i.e. only a part of the overall light emission is registered, whereas the laser emits collimated rectilinearly propagating radiation, i.e. the detector records all of it. Second, the efficiency of light emission does change between LED and laser regime resulting in different slopes, too. In our case, the detector was as close as possible to the LED such that the slope differences are dominated by the change of efficiency of light emission.

We investigated diodes from different manufacturers with threshold currents varying from $10 \mathrm{~mA}$ to well above $30 \mathrm{~mA}$. The diodes act as LED emitters below and as laser devices above threshold. Using I(U) and P(I) curves, each set of voltage and current values can be precisely attributed to either LED or laser operation. The following measurements were all made for either well defined LED, threshold region, or laser mode operation.

As all investigated diodes were inexpensive, design and production efforts are not targeting high efficiencies. For the diode shown in Fig. 2 with optical output power of $1 \mathrm{~mW}$ at around $13 \mathrm{~mA}$ and $3.4 \mathrm{~V}$, we find a conversion efficiency of only around $2.3 \%$. In this case, this means that for $1 \mathrm{~mW}$ optical output power slightly more than $40 \mathrm{~mW}$ of electrical power is dissipated. In consequence, the laser diode heats up quickly with its whole assembly by several K.

\section{MEASUREMENTS OF THE SPATIAL DISTRIBUTION OF EMITTED RADIATION}

Within a typical index guided diode laser, light propagates in the rectangular active zone channel with typical length between $200 \mu \mathrm{m}$ and $400 \mu \mathrm{m}$ and lateral dimensions varying between $1 \mu \mathrm{m}$ to $2 \mu \mathrm{m}$ by $3 \mu \mathrm{m}$ to $10 \mu \mathrm{m}$. Consequently, the emission area of the active zone of a diode laser has usually a rectangular geometry with an aspect ratio between 2 to 4 .

The spatial distribution of the emitted light may therefore - to first order - be described as being due to a plane wave passing through a respective rectangular aperture. This physics problem is well-known. Its solution allows to estimate the divergence angles once the dimensions of the active zone are known. The respective measurements are easily performed. 
Most commercial laser diodes are already sold with integrated lens in front of the laser. Therefore, we decided that students should measure both, first the far field pattern of the emitted radiation from the complete assembly, which is relevant for the direct use of such diodes and second the field patterns with lens cap removed. The latter aims to better characterize the emission characteristics of the bare laser chip.

The spatial distributions of the emitted radiation are visualized and quantitatively evaluated by directing them perpendicularly onto a sheet of paper with a $\mathrm{mm}$ grid scale. Photos of the irradiated paper are recorded with a DSLR camera in manual mode oriented as close to the optical axis as possible, i.e. its axis is also about perpendicular to the paper. Usually, exposure series are recorded for otherwise unchanged conditions (sensitivity, F stop, focal length, distance). This allows on the one hand to have long exposure images showing the grid scale for quantitative measurements although the laser spot positions are then saturated, on the other hand, the short exposure images will not show the grid but allow to analyze the non-saturated laser spot profiles.

\subsection{Far field beam profile from laser with lens cap}

Students will begin their experiments using the complete commercial laser diode, i.e. including the lens in front of the laser. Fig. 3 shows a long exposure example of the laser beam profile in $4 \mathrm{~m}$ distance from the diode. A similar profile was also measured at a smaller distance of $0.5 \mathrm{~m}$. Both measurements can be used to evaluate the beam divergence of the laser with optics.

First of all, it is obvious, that the beam quality is poor. Whereas at short distance one only observes a single nearly elliptical shape, it turns into multiple spots, which are laterally shifted by up to $1 \mathrm{mrad}$. Overall this leads to rather similar typical divergence angles in two perpendicular directions of around $1 \mathrm{mrad}$ to $1.5 \mathrm{mrad}$.

Around the central spots is a "halo" of structured stray light of much lower irradiance which covers an angular range of up to $10 \mathrm{mrad}$.

The main spot divergence angles are small and justify the use of such lasers in applications where accurate pointing is required, e.g. laser pointers, if beam quality is not as important. Students are usually familiar with these results as they know other lasers like HeNe type with similar divergence angles. Time permitting, we suggest to optionally provide a $\mathrm{HeNe}$ laser such that students may directly compare the photographically recorded beam profiles and discuss similarities (divergence angles) but also differences (beam quality). Here, we do not want to deepen a discussion on beam quality [2], which itself is an additional topic for a lab course.

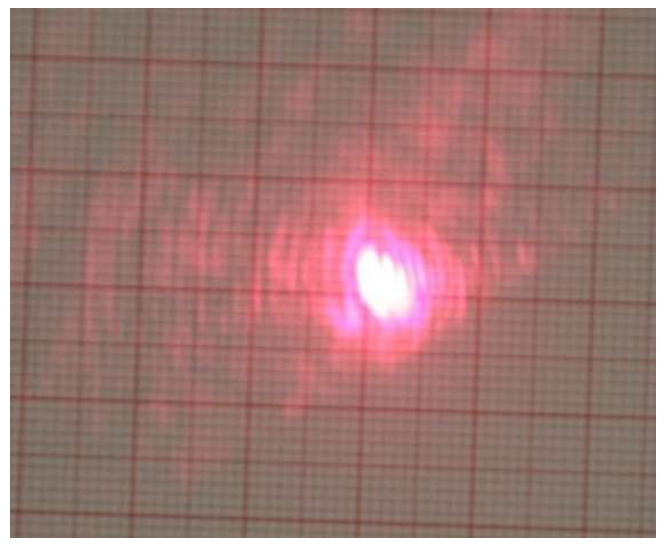

Figure 3. Far field beam pattern of red laser diode with lens cap in $4 \mathrm{~m}$ distance. At least three laterally shifted identical elliptical shape spots of around $6.5 \mathrm{~mm} \times 2 \mathrm{~mm}$ may be observed, resulting in a main spot of size $6.5 \mathrm{~mm} \times 4 \mathrm{~mm}$.

\subsection{Beam profiles from laser chip with lens removed}

As second step, the students are now required to remove the lens cap in order to measure the spatial distribution of emitted radiation directly from the laser chip. Here the low cost of the diodes allows that students and not the instructor remove 
the lens. It is kind of a win-win situation: the instructor would feel uncomfortable if the laser would be expensive and the students do feel a lower psychological barrier to manipulate the laser (not so much at risk). Still they should be careful not to damage the laser upon removal of the lens.

Fig. 4 depicts the recorded beam profiles from the diode for LED (a) and laser (b) operation (the distance of diode to screen was $11 \mathrm{~cm}$ ). One easily observes the very pronounced expected elliptical shape of the beam profile for laser operation with an aspect ratio of about four whereas the LED leads to a much more homogeneous irradiation of the screen.
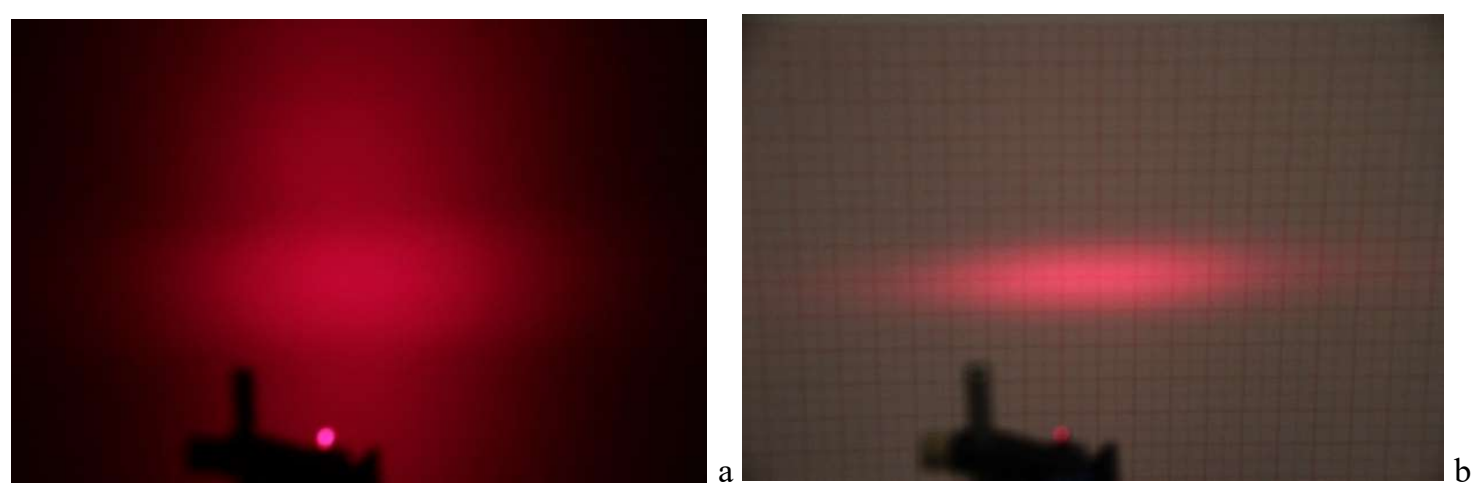

Figure 4. Spatial beam profile of red laser diode (no lens) in $0.11 \mathrm{~m}$ distance for LED (a) and laser (b) operation. Described as isotropic, the LED beam profile has a diameter of around $9 \mathrm{~cm}$, whereas the laser leads to a profile of roughly $8 \mathrm{~cm} \times 2 \mathrm{~cm}$. Due to the strongly differing output power and collimation, the exposure times varied by a factor of 40 for approximately similar total signals.

A quantitative analysis may be done by drawing line plots in the (unsaturated) images of the laser beam profile. Fig. 5 depicts results of such an analysis (using python) for the photos of Fig. 4. The normalized data in Figs. c, d refer to the red channel brightness values along the white lines shown in Figs. a and $b$. The blue contours denote lines of equal brightness of $80 \%, 60 \%$ and $40 \%$ of the maximum value. For easier analysis of Fig. $5 \mathrm{~b}$, a background subtraction was performed which also led to the artefact of the dark tripod at the lower side of the image.

From the estimated vertical and horizontal FWHM beam profile dimensions and the distance of $11 \mathrm{~cm}$ from LED/laser diode from the screen we can evaluate the respective total divergence angles. They are $\alpha_{\text {LED }} \approx 47^{\circ}$ horizontally and $\beta_{\text {LED }}$ $\approx 69^{\circ}$ vertically for the LED and $\alpha_{\text {Laser }} \approx 41^{\circ}$ horizontally and $\beta_{\text {Laser }} \approx 10^{\circ}$ vertically for the laser (orientation defined with respect to photo).

To zeroth order we interpret the laser profile as being due to simple Fraunhofer diffraction of parallel light (in active zone channel) passing through a rectangular aperture of horizontal and vertical dimensions $d_{h}$ and $d_{v}$. Typically, one uses the angle $\varphi$ of the first minimum as characteristic number which shall resemble half of the divergence angle from above. This allows to estimate the dimension $d$ of the aperture, i.e. the dimension of the emitting area on the laser chip via $d=\lambda / \sin \varphi$.

Using $\lambda \approx 650 \mathrm{~nm}$ and the above values for the laser angles we find $d_{\alpha / 2}=\lambda / \sin (\alpha / 2) \approx 1.9 \mu \mathrm{m}$ and $\mathrm{g}_{\beta / 2}=\lambda / \sin (\beta / 2)=7.5 \mu \mathrm{m}$. These values are very reasonable for semiconductor diode laser active zone dimensions. Hence, students estimate quite reasonable values for the dimensions of the active zone.

The LED also shows a horizontally elongated structure (same orientation as laser profile) but a diffuse vertically spread irradiation is superimposed. In combination this leads to a beam profile which in a rough zero order approximation could be interpreted to be more or less isotropic as expected for an LED. 

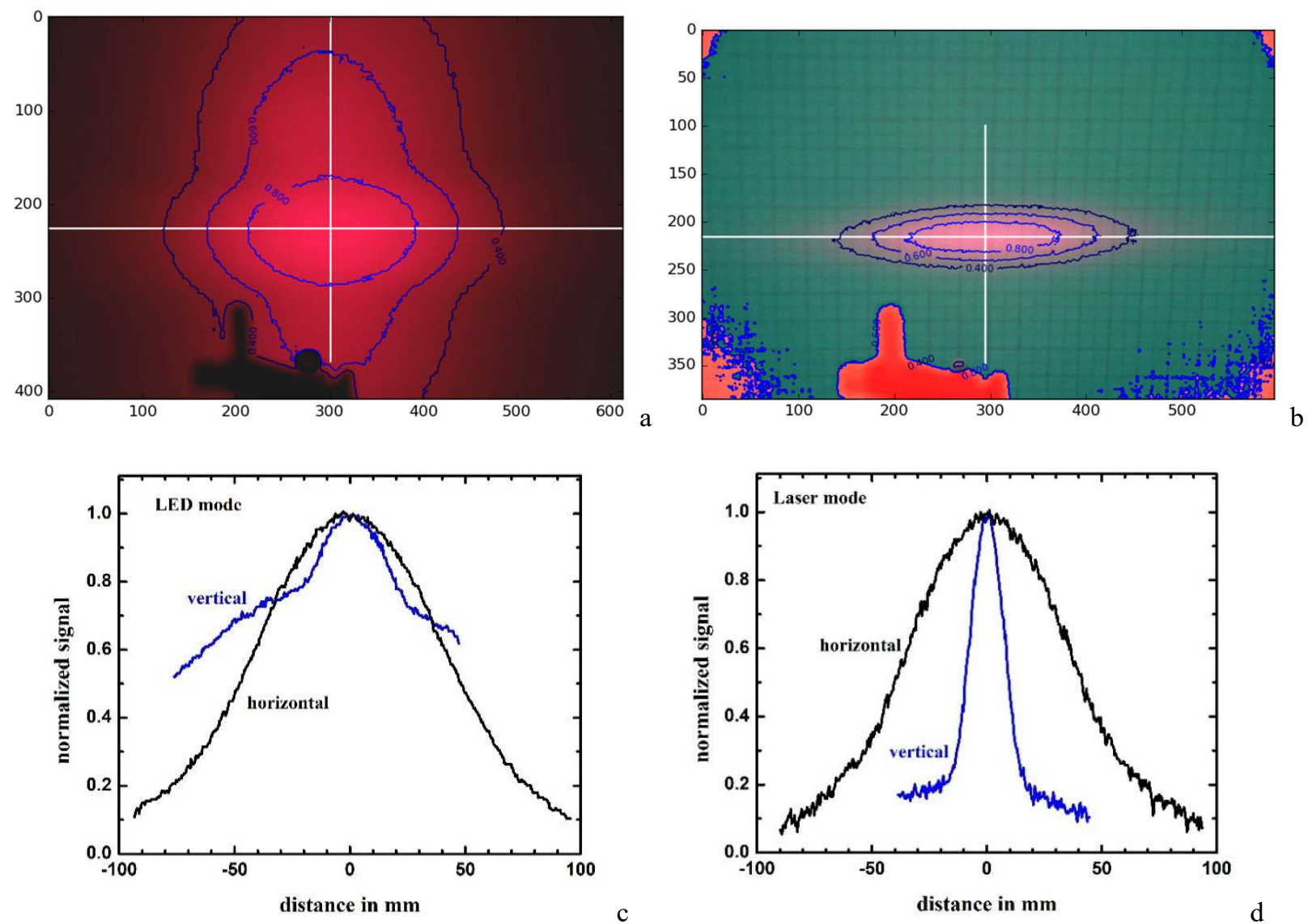

Figure 5. a,b) Pixel plot of the beam profiles of Fig.4 with contours (blue) and lines (white) for the quantitative plots (c) and (d). The FWHM of the plots are about $150 \mathrm{~mm}$ (vertical) by $95 \mathrm{~mm}$ horizontal (LED) and $19.1 \mathrm{~mm}$ (vertical) by $81.7 \mathrm{~mm}$ horizontal (Laser).

\section{SHORT SUMMARY OF TYPICAL MICROSCOPY AND SPECTROSCOPY RESULTS}

Using optical or electron microscopy, students also measure the three geometric dimensions of the rectangular laser chips. We used e.g. a Keyence VHX 600D microscope with magnification of up to 2500. Fig. 6 depicts the actual laser chip of the diode laser. Light emission is horizontally towards the left. The central more yellowish colored rectangle is a metallic contact layer to which an external contact is bonded. The horizontal dimension is the cavity length. Using a stage micrometer with known $100 \mu \mathrm{m}$ intervals, the cavity length is estimated to be $250 \mu \mathrm{m}$ and the width to be $200 \mu \mathrm{m}$. We also rotated the laser assembly and measured the third dimension of the front face of the chip to be around $100 \mu \mathrm{m}$.

Fig. 7 depicts the same diode laser observed during operation and under additional white light illumination from above. Above threshold for LED operation, the diode lights up at both facets. However, in addition, two about $20 \mu \mathrm{m}$ wide strips along the full length of the cavity also light up at the top and bottom. For increasing voltage, the diode switches to the laser mode. This manifests itself in a strongly increased scattered light from the front and back facets while simultaneously the two strips are getting brighter. More details, see [3]. 


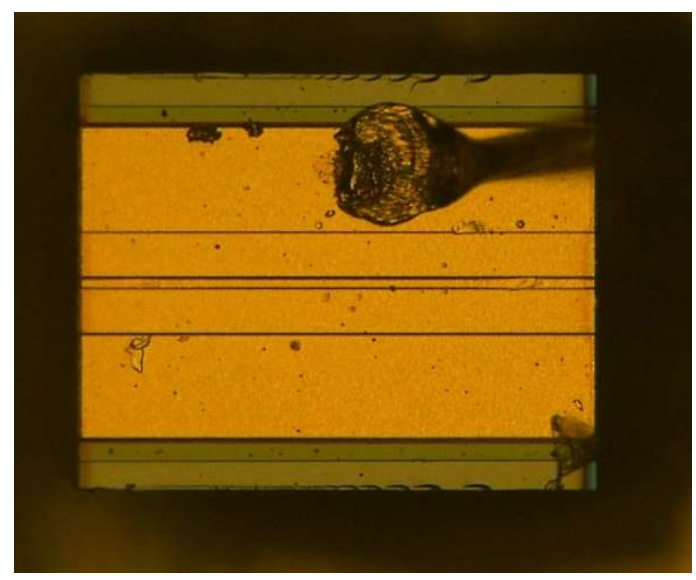

Figure 6. The diode laser semiconductor chip with length of $250 \mu \mathrm{m}$ and width of $200 \mu \mathrm{m}$.

The visible diode lasers in this study are emitting light at around $650 \mathrm{~nm}$. Semiconductor diode lasers usually have rather broad spontaneous emission spectra with respect to longitudinal mode separation. For typical laser cavity lengths below 1 $\mathrm{mm}$, expected longitudinal mode spacings are expected in the range of $\Delta \lambda=0.1 \mathrm{~nm}$ which is difficult to resolve with grating spectrometers.

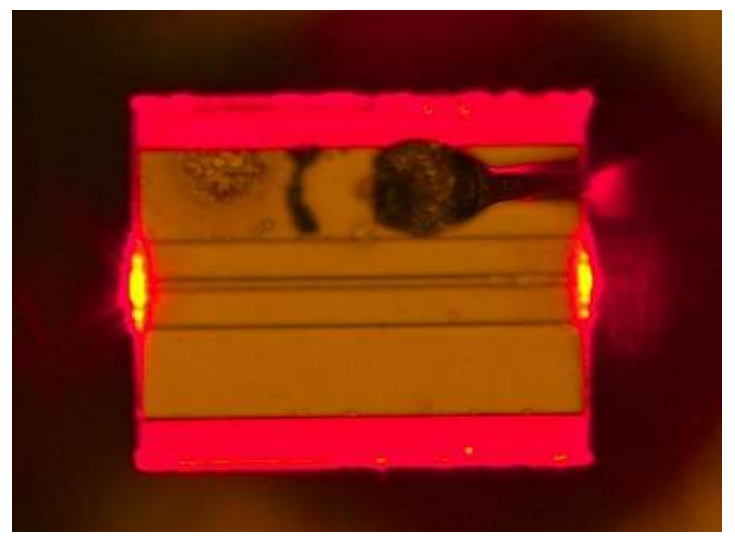

Figure 7. Microscopy image of another diode in laser mode.

We utilize our research spectrometer, a Fourier transform VIS and IR spectrometer (Bruker Vertex 80V) which allows for light to be coupled into the instrument through an external port. This constitutes a typical example of utilizing modern research equipment in lab courses, other examples are described in [4]. Fig. 8 depicts one example spectrum in the threshold region. The envelope shape of the spectrum with peak wavelength of around $653 \mathrm{~nm}$ and full width at half maximum of about $1.5 \mathrm{~nm}$ resembles the usable wavelength range. One can readily see a large number of longitudinal modes and it is possible to correlate the observed spacing with theoretical expectations based on the cavity length [3]. 


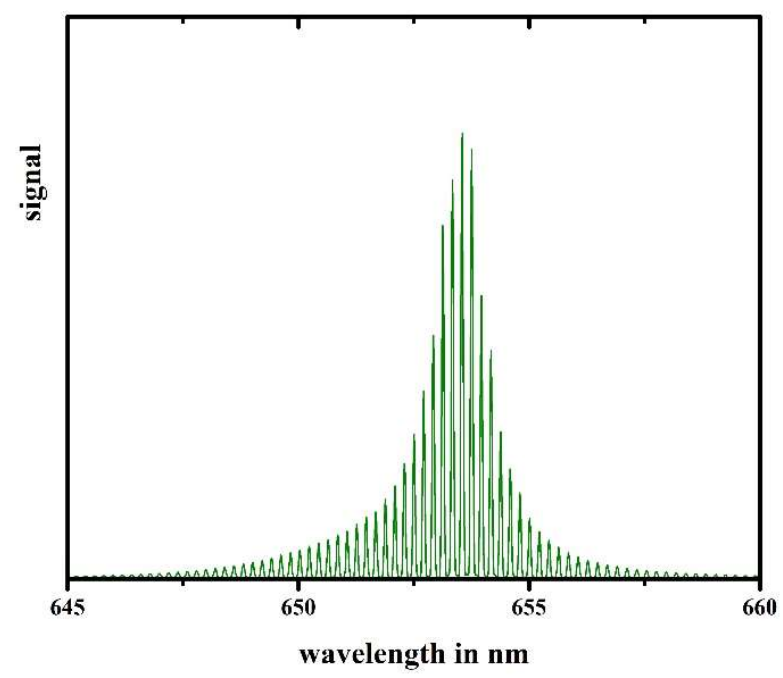

Figure 8. Emission spectrum in threshold region showing many longitudinal modes.

\section{CONCLUSIONS}

In this paper, we have described part of a laboratory course for master students of photonics. Diode lasers are investigated regarding their electrical characterization and their beam profiles. In addition we presented first results for microscopy and spectroscopy of these lasers. Altogether these lab experiments for students constitute a comprehensive investigation of these important photonics components.

\section{REFERENCES}

[1] Hooker, S and Webb, C., [Laser Physics], Oxford UP, (2010)

[2] H.J. Eichler, H.J., Eichler, J. and Lux, O., [Lasers - Basics, Advances and Applications], Springer (2018)

[3] Möllmann, K.-P., Regehly, M. and Vollmer, M., "Microscopy and spectroscopy of semiconductor lasers in lab courses", submitted

[4] Möllmann, K.-P. and Vollmer, M., "Teaching Fourier Transform Infrared Spectroscopy in physics lab courses", Eur. J. Phys. 34/6, S123-37 (2013) 